\title{
Entre Atlantique et Méditerranée : la circulation des faïences portugaises au XVII siècle en France méridionale à partir du site subaquatique de Villefranche-sur-Mer (Alpes-Maritimes).
}

\author{
Gaëlle DIEULEFET*, André TEIXEIRA**, Joana TORRES***
}

Encore inconnue dans les contextes archéologiques français, des faïences portugaises ont été récemment identifiées en Provence. C'est notamment dans un contexte portuaire, celui de Villefranche-sur-Mer, qu'ont été mises au jour plusieurs formes ouvertes correspondant principalement à des bols, des assiettes et des plats. L'étude de ces artéfacts et sa confrontation avec d'autres sites comparatifs à l'étranger permettent d'en préciser la chronologie, les lieux de productions ainsi que les principales vaisselles portugaises diffusées par la voie maritime. Leur découverte fournit de plus des indicateurs précieux sur les relations maritimes entre la France Méridionale et le Portugal durant l'époque moderne.

Mots-clés : Portugal, Provence, faïence, relations maritimes, mouillage, archéologie sous-marine, céramologie

La découverte de céramique portugaise en France Méridionale est une nouveauté. Cette provenance n'avait en effet jamais été identifiée dans les contextes archéologiques terrestres et maritimes. C'est en rade de Villefranche-sur-Mer, dans les Alpes-Maritimes (06), que sont apparus les premiers indices matériels de circulation de céramiques portugaises. Récemment reconnues et identifiées, ces productions ont été pendant plusieurs années attribuées à l'Italie, grand centre producteur de faïence. Cependant, l'avancée de la recherche en matière de céramologie permet aujourd'hui de confirmer l'origine portugaise de ces céramiques qui est aujourd'hui bien renseignée grâce aux études archéologiques menées dans le territoire portugais et ses anciennes colonies.

\section{LES INDICES MATÉRIELS DÉCOUVERTS \\ EN PROVENCE ORIENTALE : \\ LE CONTEXTE ARCHÉOLOGIQUE PORTUAIRE DE VILLEFRANCHE-SUR-MER}

Jusqu'alors inconnues des contextes archéologiques maritimes (Amouric, Richez, Vallauri 1999), c'est en Provence orientale que nous retrouvons actuellement le plus important corpus de céramiques portugaises (Dieulefet 2013, 245). Ces attestations matérielles proviennent principalement du domaine maritime et notamment de la rade de Villefranche-sur-Mer. Cette rade, qui fait l'objet depuis plusieurs années d'opérations archéologiques sous-marines, fournit de nombreux vestiges liés à l'activité portuaire.

\section{Un vaste port ouvert sur la Méditerranée}

Les dépotoirs portuaires sont porteurs de nombreuses informations, en raison de leurs spécificités, tant au niveau de l'analyse des échanges qu'au niveau de la compréhension du commerce et de la vie des sociétés maritimes (Dieulefet 2015, 207). En effet, lieu de vie et de circulation, les ports ont accueilli en tout temps des navires venant d'horizons très divers. Ces navires de guerre, de commerce, de pêche ou de cabotage ont laissé des traces dans le paysage sous-marin, avant tout d'ordre matériel. En rade de Villefranche-sur-Mer, ces attestations sont multiples et quantitativement importantes. En effet, la présence du mont Vinaigrier au nord, du mont Boron et du mont Alban à l'ouest, ainsi que de la presqu'île de Saint-Jean-Cap-Ferrat à l'est, offre une protection à la rade. Idéalement abritée du Sirocco (1), elle ne subit que légèrement la Tramontane (2) durant l'hiver. Seul le Libeccio (3), vent redouté par les navigateurs méditerranéens, peut pénétrer dans la rade

\footnotetext{
* Docteure en Archéologie d'Aix-Marseille Université, chercheuse associée au LA3M UMR-CNRS 7298

** Professeur à la Faculdade de Ciências Sociais e Humanas - Universidade Nova de Lisboa ; coordinateur du groupe d'archéologie du CHAM (Portuguese Centre for Global History)

*** Doctorante à la Faculdade de Ciências Sociais e Humanas - Universidade Nova de Lisboa ; chercheuse du groupe d'archéologie du CHAM (Portuguese Centre for Global History)

${ }^{1}$ Le Sirocco est un vent saharien de secteur est et sud-est dominant

${ }^{2}$ La Tramontane est un vent de secteur nord.

${ }^{3}$ Le Libeccio est un vent de secteur sud-ouest
} 
(Gioffredo 2008, 134). Néanmoins, avec ses grandes profondeurs qui varient de 17 à 95 mètres et un espace de navigation vaste, la rade peut aisément accueillir de nombreux navires à fort tirant d'eau.

Depuis 1338, la cité de Villefranche est dépendante du Duché de Savoie. Exploitant les qualités naturelles de la rade, ce n'est qu'avec l'établissement de la citadelle Saint-Elme à partir de 1554, puis le transfert de la capitale des États de Savoie de Chambéry à Turin en 1563 que seront mis en œuvre les premiers aménagements portuaires. Par la suite, la mise en place du port franc en 1612 et du lazaret pour la quarantaine en 1650 (Lacroix, Bracq, Reymonenq 1999, 95) vont permettre l'accroissement de la fréquentation de cet espace portuaire qui achève sa longue mutation à la fin du $\mathrm{XVII}^{\mathrm{e}}$ siècle et prend place dans le réseau portuaire régi par Marseille et Toulon (fig. 1).

\section{La recherche archéologique sous-marine}

Les découvertes en 1979 et 1985 de deux épaves majeures, la Lomellina (1516) puis la Sainte-Dorothéa (1693), ont mis en évidence le potentiel archéologique de ce site. Les opérations de prospections menées à partir de 1991 ont permis d'enrichir la carte archéologique de la rade par l'identification de quatre autres gisements en lien avec le port: la Batterie des Deux-Rubes, le Palais de la Marine, la Citadelle et la Quarantaine (fig. 2) (4). Pour l'heure, les découvertes de céramiques portugaises proviennent essentiellement de la partie nord-ouest de la rade dans deux gisements principaux : le Palais de la Marine et la Citadelle.

Le gisement du Palais de la Marine se situe à la lisière d'un herbier de posidonie et d'un tombant vaseux qui commence à 11 mètres de profondeur. Le mobilier

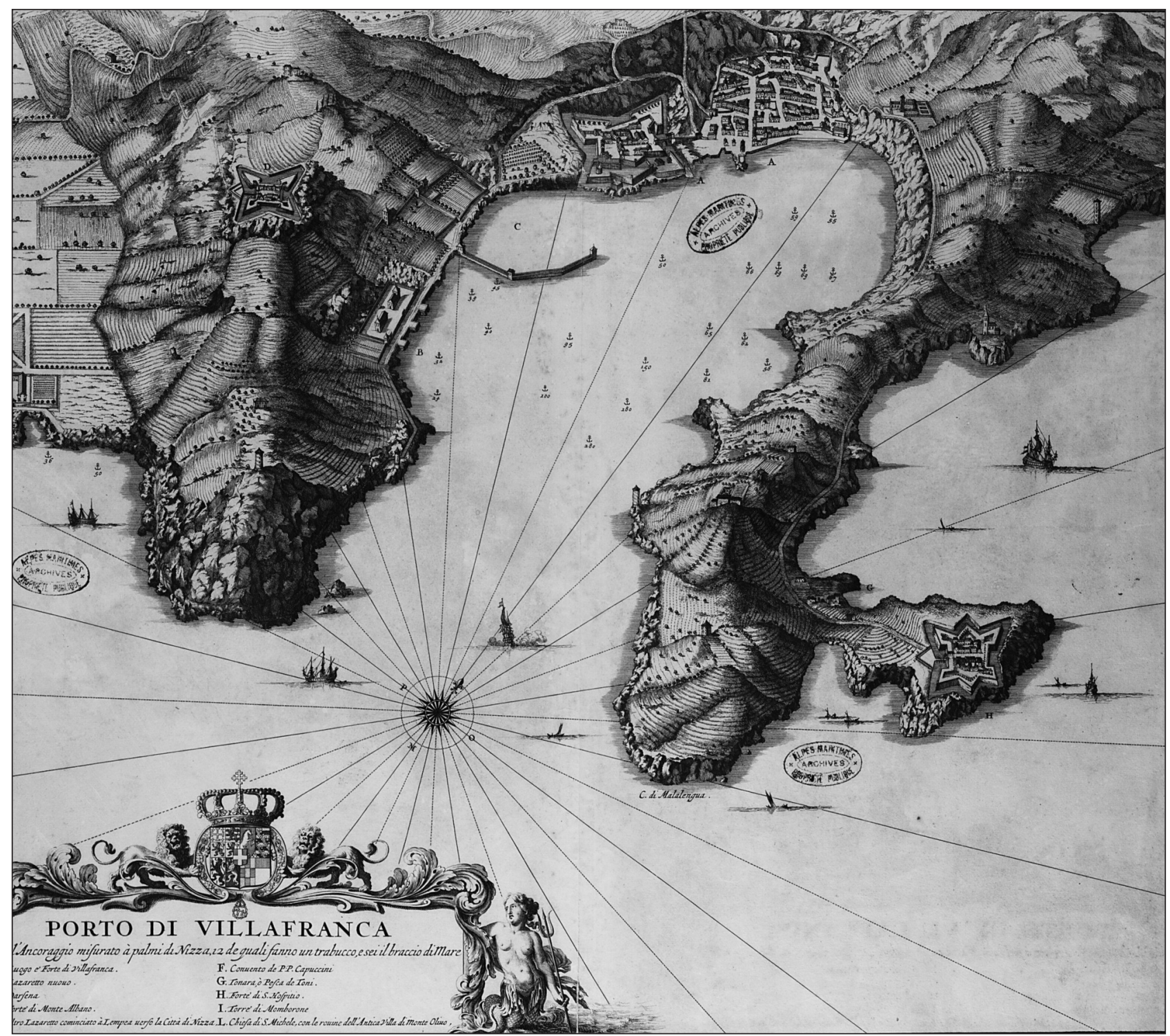

Fig. 1 : Porto di Villafranca, archives des Alpes-Maritimes (réf : 01FI-0046) XVIII ${ }^{e}$ siècle.

\footnotetext{
${ }^{4}$ Prospections menées par l'Association « Anao, L'aventure sous-marine » sous la direction de Éric Dulière. L'ensemble du mobilier mis au jour est aujourd'hui conservé au Musée de la Préhistoire Régionale de Menton.
} 


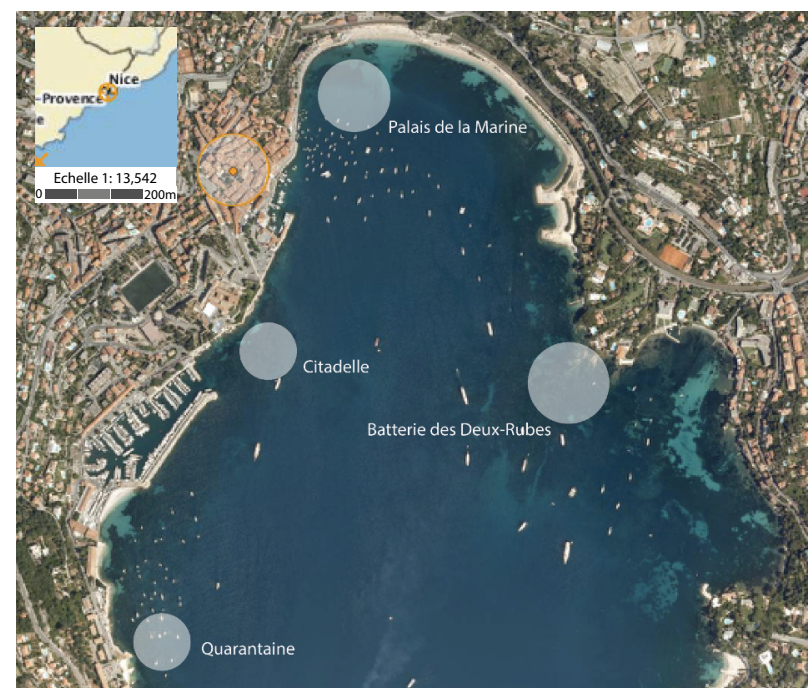

Fig. 2 : Localisation des secteurs prospectés dans la partie nord de la rade.

daté majoritairement entre le XVII ${ }^{\mathrm{e}}$ et le $\mathrm{XVIII}{ }^{\mathrm{e}}$ siècle est présent dans une couche sablo-vaseuse de 10 à 30 centimètres d'épaisseur. La formation de ce dépotoir est en lien avec une zone de mouillage naturellement présente face à la ville et fréquentée par les caboteurs, les commerçants ou encore les pêcheurs. Le gisement de la Citadelle, au pied du fort Saint-Elme, commence au départ d'un plateau sableux à 12 mètres de profondeur. Le mobilier est daté principalement du $\mathrm{XVI}^{\mathrm{e}}$ et du $\mathrm{XVII}^{\mathrm{e}}$ siècle. Contrairement à celle du dépotoir du Palais de la Marine, sa formation est en lien avec une zone de mouillage qui, protégée par les canons du fort, est surtout fréquentée par les galères ducales et celles des alliés du Duché de Savoie (Dieulefet, Dulière 2015, 53).

Le contexte de dépôt de ces céramiques résulte ici d'accumulations successives de mobilier provenant soit d'une perte accidentelle soit d'un rejet par-dessus bord (Dieulefet 2013, 403). D'autre part, dans le cas de Villefranche-sur-Mer, il s'agit plus vraisemblablement de rejets (suite à la casse d'un objet ou d'un tri par exemple) que d'une perte de cargaison comme celle de Brescou 1 à Agde (Pomey et al. 1987-1988, 52). Le mobilier ne forme pas une concentration compacte et homogène, mais un ensemble typologiquement hétérogène qui conserve bien souvent des marques d'usage et d'appropriation. C'est le cas des céramiques portugaises, découvertes parfois à plusieurs dizaines de mètres l'une de l'autre. De par la nature d'un dépotoir portuaire, les céramiques portugaises sont associées à bien d'autres productions. En provenance d'Italie, d'Espagne, de Hollande, de Turquie, de Provence ou encore de Chine, la diversité des attestations matérielles donne une image des grandes productions qui circulent par la voie maritime. D'autres matériaux complètent cette culture matérielle maritime avec la mise au jour d'artefacts en verre, en bois, en métal, ainsi que de nombreuses pipes à tabac et des restes fauniques liés à l'alimentation des équi- pages (Dieulefet, Dulière 2011, 48). Actuellement, les céramiques portugaises sont essentiellement représentées par des formes ouvertes en faïences. Ce corpus, qui regroupe actuellement 31 NMI (5), fait exception aux regards des grands ensembles céramiques italiens, espagnoles ou encore provençaux découverts à Villefranchesur-Mer.

\section{LES FAÏENCES PORTUGAISES DE VILLEFRANCHE-SUR-MER}

La présence à Lisbonne d'ateliers de production de céramique émaillée blanche est documentée dès le milieu du $\mathrm{XVI}^{\mathrm{e}}$ siècle. La référence la plus ancienne, datée de 1561, concerne une procédure inquisitoire contre João de Góis, " oleyro de malaga e azulejos», originaire d'Anvers (Torres 2011, 52 ; 126). Celui-ci, quatre ans plus tard, est mentionné dans un livre d'impôt de Lisbonne en tant que "mestre de malega branca» selon Vergílio Correia (Sebastian 2011, 560). Mentionné en tant qu'habitant de cette ville depuis sept ou huit ans, son activité pourrait remonter aux années 1550 (Torres 2011, 52 ; 132), mais nous n'en avons actuellement aucune trace archéologique. Toutefois, au cours de ce même siècle, d'autres centres de production se sont développés, tels que Coimbra et Vila Nova, atteignant une large diffusion au cours du XVII ${ }^{\mathrm{e}}$ siècle au Portugal et dans ses comptoirs commerciaux.

\section{Description des formes}

L'ensemble des céramiques de Villefranche-sur-Mer appartient à cette production portugaise. Elle se caractérise, d'une façon générale, par son revêtement extérieur en glaçure stannifère blanche épaisse et brillante (quelques fois modifiée par les conditions de dépôt), présentant parfois de la décoration peinte en bleu de cobalt et/ou brun de manganèse. Les pâtes ont une tonalité claire, entre le jaune et le beige, avec une texture compacte à tendance crayeuse, caractéristique de la région de Lisbonne (Sebastian 2010, 211). Quatre types formels ont été identifiés : des tigelas (bols), des covilhetes (plats tronconiques), des pratos covos (assiettes) et des pratos fundos (plats), terminologie consacrée dans les travaux de Raphael Bluteau (1712-1728) et dont la collecte de données a commencé en 1680 .

Les bols (type 1) sont représentés par trois exemplaires avec de légères différences au niveau du diamètre, de l'épaisseur de la paroi et de la présence d'une carène plus ou moins prononcée. Ainsi, les bols $1 \mathrm{~b}, 1 \mathrm{c}$ et $1 \mathrm{a}$ ont respectivement un diamètre d'ouverture de $15,14.5$ et $13 \mathrm{~cm}$ et à la base de $6.5,6$ et $4 \mathrm{~cm}$. Le fond est marqué par une atténuation de la carène qui s'abaisse progressivement selon ses trois variantes typologiques (fig. 3.1).

Les plats tronconiques (type 2) présentent un bord légèrement déversé et arrondi de section semi-circulaire

\footnotetext{
${ }^{5}$ Nombre Minimum d'Individus.
} 
et un fond annulaire relativement bas. Ils se caractérisent par un diamètre d'ouverture, de 14 et $16 \mathrm{~cm}$, peu supérieur au diamètre du pied de 10,5 et $12 \mathrm{~cm}$ (fig. 3.2).

Les assiettes (type 3), malgré de légères différences entre elles, semblent suivre le même genre de moule (Sebastian 2010, 252-273), comme en témoigne leur section. Reposant sur un pied annulaire, elles sont munies de bords semi-circulaires déversés et légèrement épaissis. Les diamètres d'ouverture des exemplaires 3a, $3 \mathrm{~b}, 3 \mathrm{c}, 3 \mathrm{~d}$ et $3 \mathrm{e}$ sont compris entre 20 et $22 \mathrm{~cm}$ et à la base entre 11 et $12 \mathrm{~cm}$, indiquant l'utilisation de la même bitola (6). Au niveau de l'épaisseur des parois et de la hauteur, seul le plat $3 \mathrm{e}$ présente des spécificités qui se caractérisent par le rétrécissement des parois et leur surélévation de $4 \mathrm{~cm}$. L'exemplaire $3 \mathrm{f}$ a globalement des dimensions supérieures, avec $26 \mathrm{~cm}$ de diamètre d'ouverture et $17 \mathrm{~cm}$ de pied (fig. 4.3).

Les plats (type 4) ont une paroi oblique et ne présentent pas de carène. Le diamètre d'ouverture, qui est uniquement donné sur le plat $4 \mathrm{a}$, est de $30 \mathrm{~cm}$ et présente un profil déversé épaissi à l'extérieur. Le pied de cet exemplaire, de $14 \mathrm{~cm}$ de diamètre, est en forme d'omphalos tandis que l'exemplaire $4 \mathrm{~b}$ repose sur un fond annulaire de $16 \mathrm{~cm}$ de diamètre (fig. 4.4).

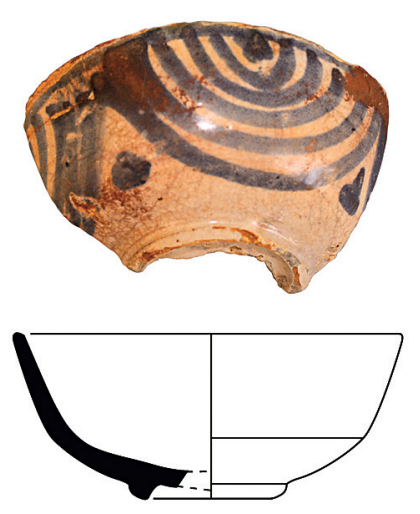

$1 \mathrm{a}$
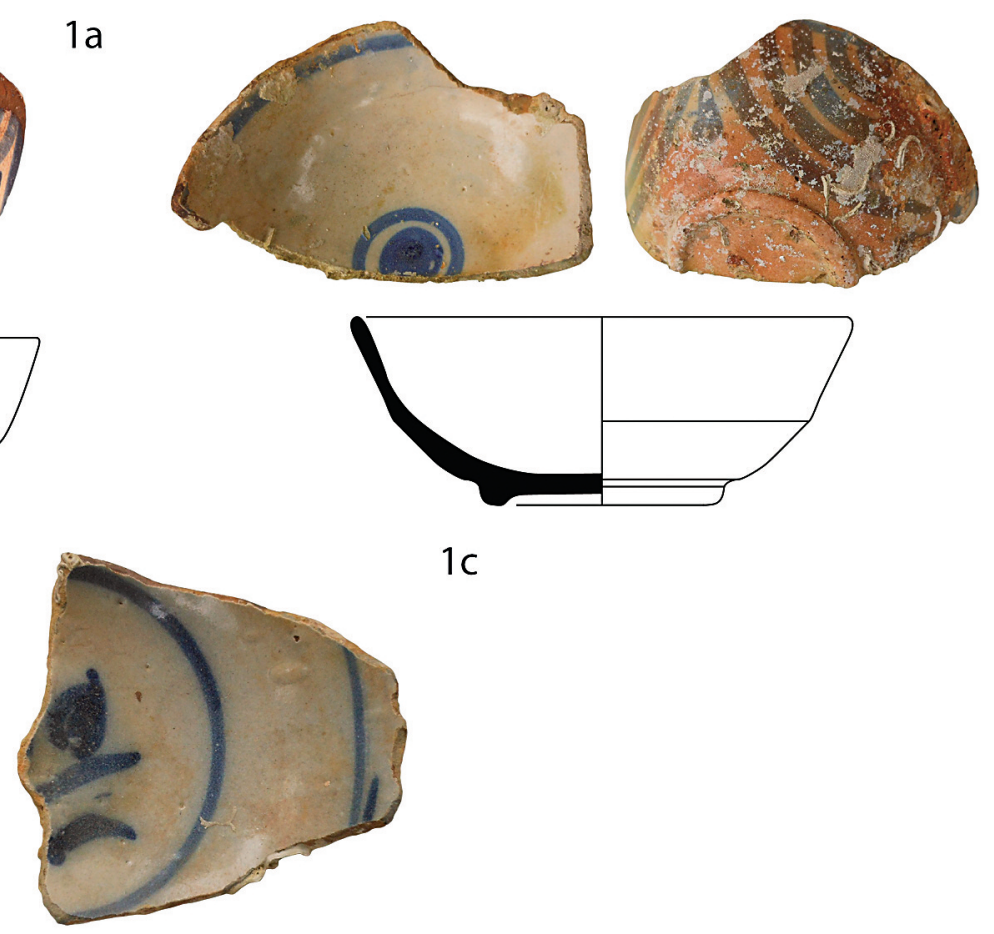

$1 c$
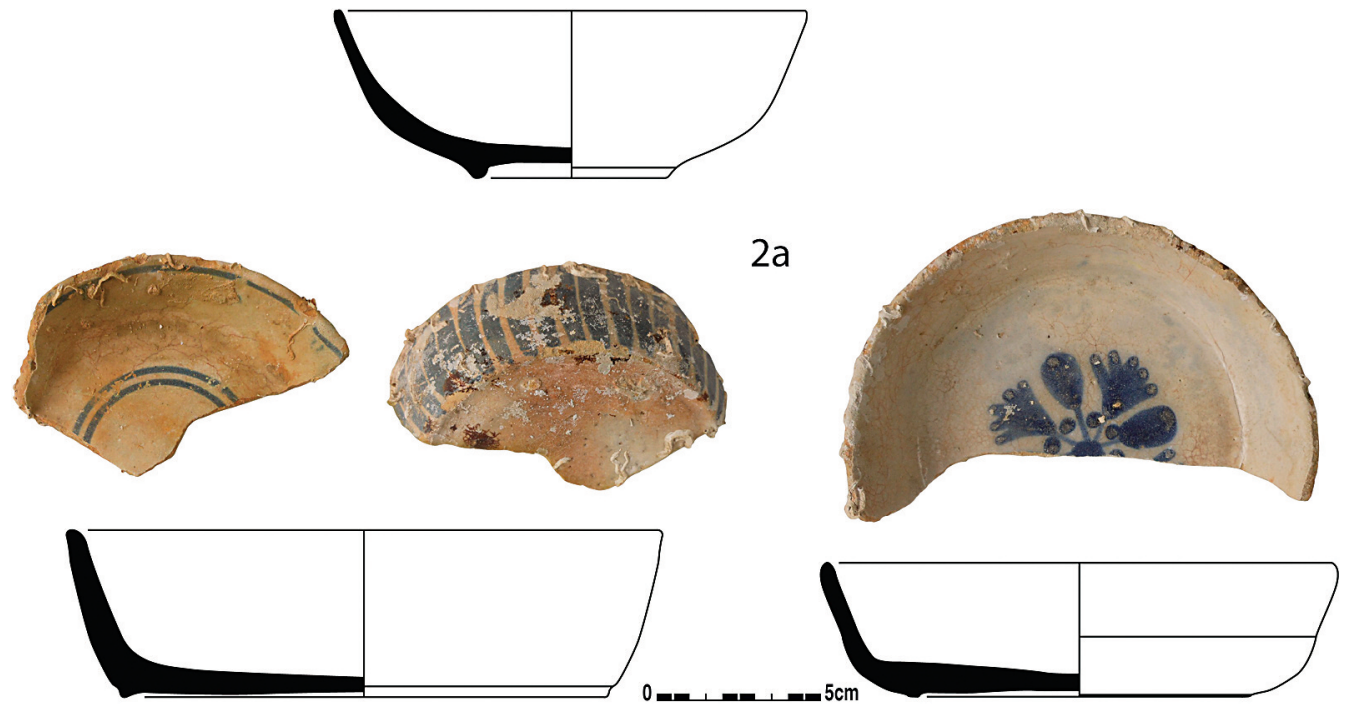

Fig. 3 : Bols et plats tronconiques en faïence blanche et décor bleu (clichés, dessins et vectorisations : G. Dieulefet).

\footnotetext{
${ }^{6}$ La bitola désigne les mesures de référence des potiers portugais afin obtenir des poteries aux dimensions identiques.
} 

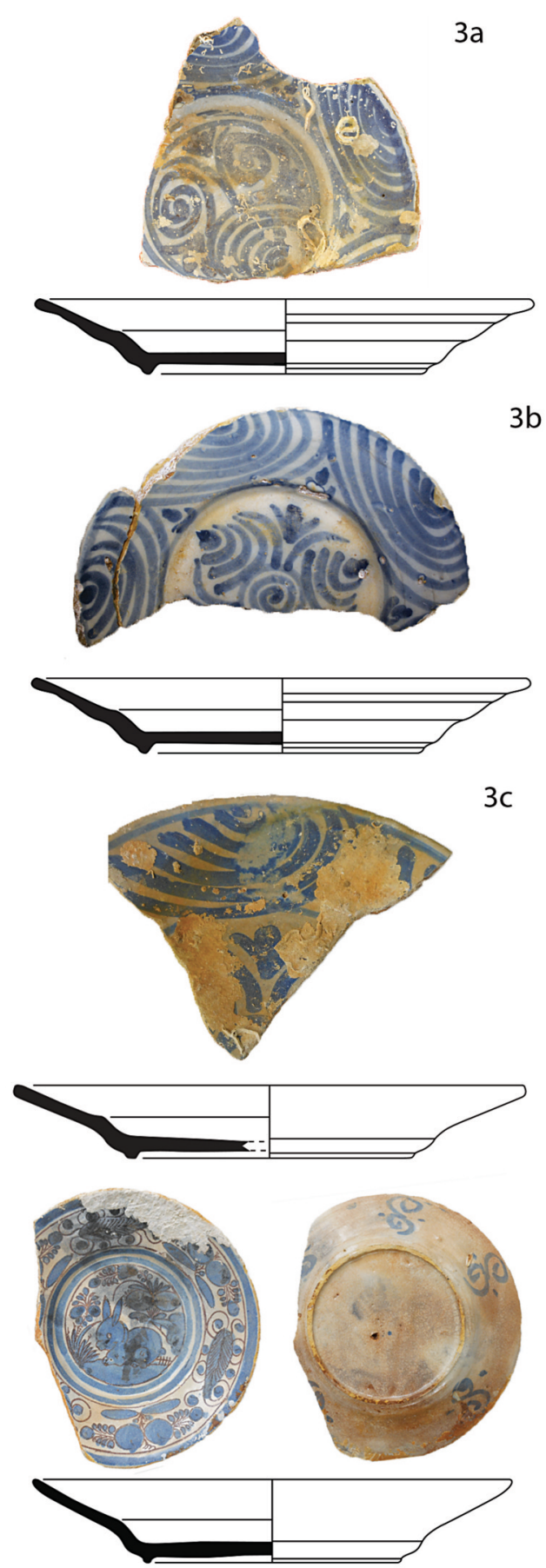

$4 a$
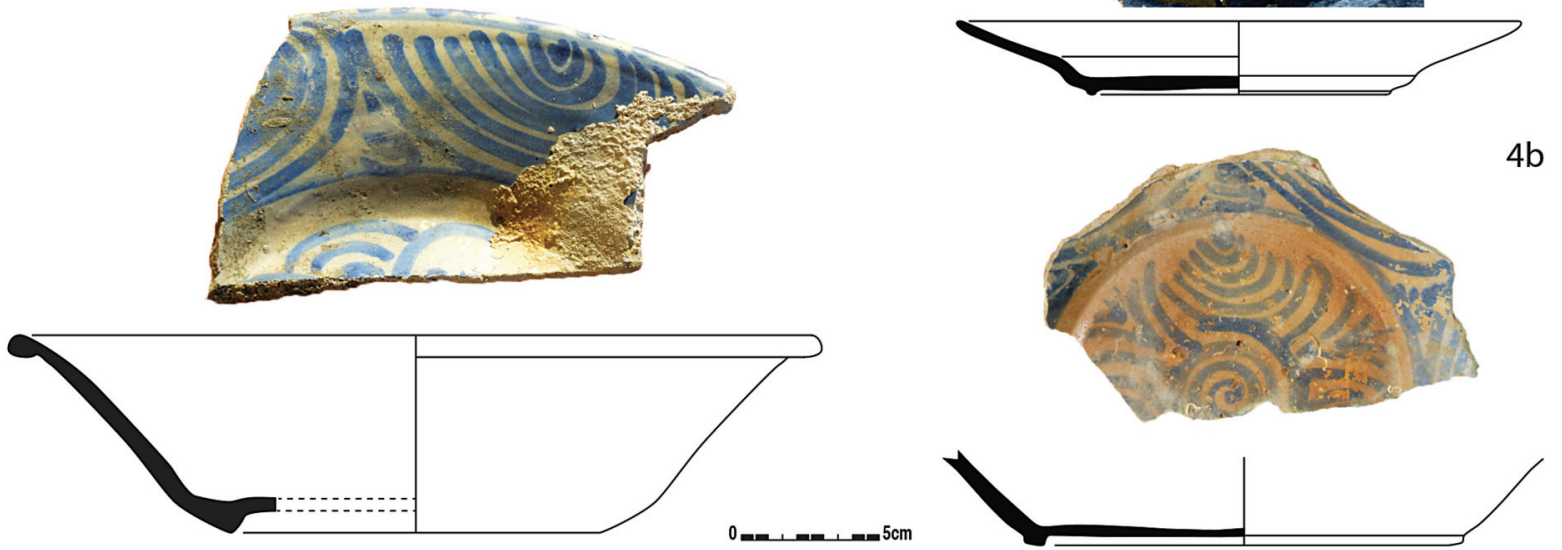

Fig. 4 : Assiettes et plats en faïence blanche et décor bleu à rehauts violet (clichés, dessins et vectorisations : G. Dieulefet).
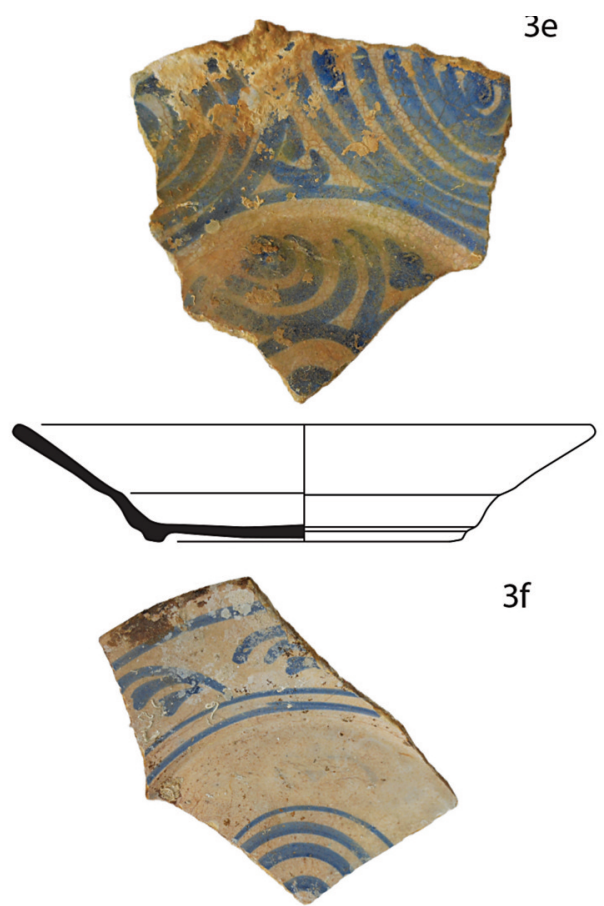

$3 f$

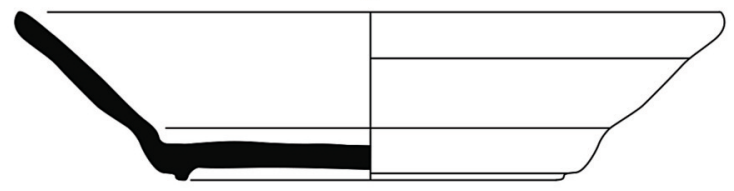

$3 g$
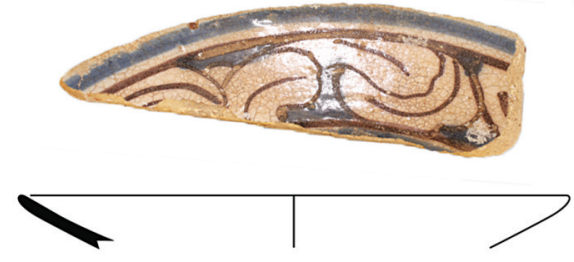

$3 \mathrm{~h}$

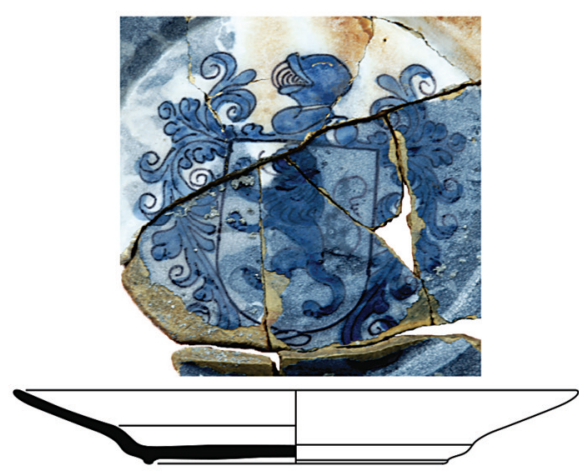

$4 b$ 
Ces quatre typologies sont associées au service de table et à une consommation alimentaire individuelle. Le bol et le plat sont utilisés pour les repas cuits et/ou les ragoûts où la présence du bouillon est significative. L'assiette est quant à elle utilisée pour contenir les aliments rôtis et frits ou pour des repas cuits sans bouillon. Les covilhetes sont généralement associés aux différents types de desserts portugais (Bluteau v.2, 594) comme les fruits confits ou le célèbre blanc-manger. De plus, les assiettes et les plats d'un diamètre supérieur à $25 \mathrm{~cm}$ sont utilisés pour servir la nourriture à table, qui est ensuite redistribuée à chaque personne. Il convient également de signaler que, dans des ensembles céramiques identiques détectés ailleurs, la répétition décorative dans des formes distinctes permet alors de composer des " services de table » comme observés à Cadix (Gutierrez et al. 2012, 271).

\section{Description des décors et sites comparatifs}

L'ensemble céramique de Villefranche-sur-Mer ne révèle pas la même richesse décorative pour toutes les formes. Les pièces $3 \mathrm{a}, 3 \mathrm{~b}, 3 \mathrm{c}, 3 \mathrm{e}, 4 \mathrm{a}, 4 \mathrm{~b}, 1 \mathrm{a}$ et $1 \mathrm{~b}$ présentent un thème décoratif de demi-cercles associés à d'autres éléments secondaires, tels que des enroulements circulaires et des motifs végétaux stylisés, toujours traités au bleu de cobalt sur fond blanc. En ce qui concerne les assiettes et les plats, les surfaces extérieures ne présentent aucune décoration. Elle est uniquement présente sur la face interne, soit sur le marli (demicercles avec sept à huit lignes parallèles), soit sur le fond (demi-cercles, enroulements au centre : $3 \mathrm{~b}, 3 \mathrm{e}$ et $4 \mathrm{~b}$; enroulements latéraux : $3 \mathrm{a}$; motifs végétaux : $3 \mathrm{a}, 3 \mathrm{~b}, 3 \mathrm{c}$, $3 e$ et $4 b)$. Les bols ont un décor externe composé de séries de six lignes parallèles de demi-cercles. L'intérieur, parfois laissé nu (1a), est plus généralement décoré d'une ligne qui souligne le bord et d'un enroulement circulaire central sur le fond (1b).

Pour leurs datations et leurs aires de diffusion, nous trouvons des similitudes entre les pièces $3 \mathrm{~b}$, 3e et $4 \mathrm{~b}$ de Villefranche-sur-Mer et le type 11 de la Rua de Buenos Aires, un important contexte de production potière du $\mathrm{XVII}^{\mathrm{e}}$ siècle à Lisbonne (Batalha et al. 2012, 958). En ce qui concerne les sites de consommation, on peut souligner les exemplaires du $\mathrm{XVII}^{\mathrm{e}}$ siècle découverts à Lisbonne (Cardoso, Batalha 2013, 123) et du Machico, dans l'île de Madère (Sousa 2011, 225), du galion Sacramento naufragé près de Salvador da Bahia en 1668 (Gomes 2011, 105), ou encore ceux de l'ancienne colonie portugaise du Sacramento en Uruguay (Fusco-Zambetogliris 2003, 56). Les marlis des assiettes 3a, 3b, 3c et 3e nous renvoient vers la même période dans les villes portugaises de Lagos (Oliveira 2010, 827), Torres Vedras (Luna, Cardoso 2006, 109), Torres Novas (Carreira 2005, 51) et Lisbonne pour le dernier quart du XVII ${ }^{e}$ siècle jusqu'au début du XVIII ${ }^{\mathrm{e}}$ siècle (Brito, Barbosa 2012, 154). De même, on peut citer les exemplaires de la colonie portugaise en Uruguay (Fusco-Zambetogliris 2003, 54) ou encore du couvent des Augustins dans la cité portugaise de Goa (Silva 2013, 189).

Pour le bol 1a, nous retrouvons des similitudes avec les exemplaires découverts à Lagos (Oliveira 2010, 828) et dans le site de Garrett-Beadle à Charlestown, (Massachusetts, États-Unis) où il a été daté entre 1640 et 1660 (Pendery 1999, 67; 71). Le bol 1b, que l'on peut rattacher également aux productions de Lisbonne de la Rua de Buenos Aires (Batalha et al. 2012, 959), apparaît également dans des sites de consommation du $\mathrm{XVII}^{\mathrm{e}}$ siècle à Lisbonne, dans le couvent de São Francisco (Torres 2011, 402), de la Casa dos Bicos (Moita 1987, 31), mais aussi dans le site de Charlestown (Pendery 1999, 67; 71). Ces sites comparatifs nous permettent de proposer une datation dans le courant de la deuxième moitié du XVII ${ }^{\mathrm{e}}$ siècle (Sebastian 2010, fig. 319). Ce décor apparait également sur d'autres typologies de la première moitié du $\mathrm{XVII}^{\mathrm{e}}$ siècle, avec des variations, à Leiria dans le centre du Portugal (Trindade 2012, 171), dans le couvent de Santa Cruz dans l'île de Madère (Sousa 2011, 224), mais aussi à Silves durant la deuxième moitié du XVII ${ }^{\mathrm{e}}$ siècle (Trindade 2009b, 255). D'autres attestations nous viennent également du château de Dublin, toujours pour la deuxième moitié du $\mathrm{XVII}^{\mathrm{e}}$ siècle (Casimiro 2010, 389), du port anglais de Plymouth (Casimiro 2011, 255) et de la colonie portugaise en Uruguay (Fusco-Zambetogliris 2003, 57) où ils sont datés entre 1600 et 1640 .

Deux autres exemplaires de Villefranche-sur-Mer, aux typologies différentes, présentent néanmoins la même gamme de décors développés en amont. Le plat tronconique $2 \mathrm{a}$ présente l'esthétique des bols $1 \mathrm{a}$ et $1 \mathrm{~b}$, bien qu'avec une composition distincte de plusieurs lignes verticales parallèles externes et deux lignes concentriques internes qui soulignent le fond et le bord. Le décor de l'assiette $3 \mathrm{f}$, qui s'écarte de l'horror vacui des autres exemplaires, répète l'enroulement circulaire central interne et porte une version simplifiée et stylisée des demi-cercles parallèles sur le marli encadré de deux lignes concentriques.

Le bol $1 \mathrm{c}$ et le plat tronconique $2 \mathrm{~b}$ de Villefranchesur-Mer portent d'autres décors toujours traités en bleu sur fond blanc. Le premier exemplaire a un décor interne composé de deux lignes concentriques soulignant le bord et le fond, qui encadrent un médaillon central à motif végétal abstrait. Ce dernier est similaire à un fond de plat mis au jour sur l'épave de la frégate portugaise Santo António de Taná, naufragée à Mombasa en 1697 (Piercy 1977, 343). Le deuxième exemplaire présente quant à lui un décor interne végétal similaire aux exemplaires datés du XVII ${ }^{e}$ siècle du couvent de São Francisco à Lisbonne (Torres 2011, 356) et de l'église de la Misericórdia de Almada (Sabrosa, Santo 1992, 8-9).

Un autre décor est représenté sur le plat $3 \mathrm{~d}$. La face externe reçoit un simple «S» peint en bleu qui contraste avec l'abondant décor interne traité en bleu et en brun de manganèse tirant sur le violet. Le marli est souligné par deux séries de lignes bleues et brunes violacées qui encadrent une frise. Celle-ci est composée de trois feuilles d'Armoise, agrémentées ici de courbes et de points formant le décor dit d'aranhões (grande araignée), et de deux pêches. Ses éléments iconographiques, notamment la feuille d'Armoise et la pêche, sont dérivés de l'orne- 
mentation de la porcelaine chinoise et sont reproduits dans les ateliers portugais uniquement pour leur attrait esthétique, sans signification symbolique (Monteiro, Pais 2003, 84-95). Au centre de l'assiette, un lapin est représenté installé dans un jardin matérialisé par une petite barrière, imitant encore une fois les fameuses représentations chinoises de lièvres (Monteiro, Pais 2003, 90). Ce type de décor est également attesté dans des sites de consommations datés du troisième quart du XVII ${ }^{\mathrm{e}}$ siècle au Portugal et dans ses territoires d'outre-mer, nous permettant ainsi de préciser sa période de diffusion. Le décor en frise du marli est par ailleurs bien renseigné grâce aux exemplaires découverts sur l'épave du Sacramento (Gomes 2011, 31), du Santo António de Taná (Piercy 1978, 304; Sasson 1981, 116) ou encore à Ponta do Leme Velho au Cap-Vert vraisemblablement de la même époque (Gomes et al. 2014, 163). On peut citer également les contextes archéologiques du couvent de São João de Tarouca au Portugal, notamment l'ensemble A.3.1.2 (Castro, Sebastian 2008, 18), et celui du collège jésuite de São Salvador, au Brésil, tous deux datés de la deuxième moitié du XVII ${ }^{\mathrm{e}}$ siècle (Etchevarne et al. 2012, 28). Enfin, certaines collections muséologiques portugaises fournissent des datations aux alentours de 1670 (Calado, Lima 2005, 131-132) ou plus largement dans le troisième quart du $\mathrm{XVII}^{\mathrm{e}}$ siècle (Monteiro, Pais 2003, 84-85).

Le fragment $3 \mathrm{~g}$, correspondant à un bord d'assiette, présente un décor interne traitée en bleu et en brun, toujours sur fond blanc. Il se compose d'une frise au décor dit «baroque » qui comporte des motifs d'acanthes détourés en brun (Queirós 2002, 60). Bien que la chronologie retenue actuellement pour ce décor est la deuxième moitié du XVII ${ }^{\mathrm{e}}$ siècle (Queirós 2002, 446; Calado 2003, 88-89, 92-93; Monteiro, Pais 2003, 102103), des analogies avec d'autres exemplaires découverts à Lisbonne, datés du milieu du XVIII ${ }^{\mathrm{e}}$ siècle, permettent d'étendre leur période de production (Mangucci 2007, 2-6). De plus, ce décor apparait sur les jarres de stockages embarquées sur le Santo António de Taná (Piercy 1977, 334).

Un seul décor armorié, toujours traité en bleu et brun sur fond blanc, est présent sur le plat $3 \mathrm{~h}$. Habituellement associé aux armes des «Silva », mais aussi d'autres lignages portugais (Monteiro, Pais, 2003, 100), sa reproduction est devenue ordinaire. Ainsi son utilisation n'était plus exclusivement véhiculée par la noblesse, ce qui a contribué à sa large diffusion. D'autres exemplaires ont été identifiés dans les villes portugaises de Porto (Sá 2012, 969) et de Torres Novas (Carreira 2005, 49), sur l'épave du Sacramento (Etchevarne, Gomes 2012, 825; Gomes 2011, 105), ou encore à Charlestown (Pendery 1999, 67 ; 70). Le même motif des années 1677, avec de légères variations, a été également identifié dans une collection privée (Queirós 2002, 52; Calado, Lima 2005, 132), ainsi que dans un contexte londonien daté entre 1640 et 1700 (Casimiro 2011, 65). Actuellement, l'apparition de ce blason est donnée aux années 1625 , bien que son contour brun-violet ne soit mentionné qu'à partir du milieu du XVII ${ }^{\mathrm{e}}$ siècle (Calado 1992, 38), et semble se prolonger jusqu'à la fin du XVII ${ }^{\mathrm{e}}$ siècle.

\section{Ateliers de production \\ et datations des formes rencontrées}

Les faïences découvertes à Villefranche-sur-Mer, malgré leur faible quantité, sont assurément originaires des ateliers de Lisbonne, attribution confirmée par l'analyse macroscopique de leurs caractéristiques technologiques et techniques telles que la pâte, la glaçure et les éléments de décor (Sebastian 2012, 947-949). La plus importante ville portugaise a eu en effet, depuis le Moyen Âge, une activité potière intense qui a laissé de nombreuses traces écrites dans les sources, ainsi que des attestations matérielles de plus en plus nombreuses (Bugalhão et al. 2009).

À l'aube du XVI ${ }^{\mathrm{e}}$ siècle, avec l'augmentation de la consommation de faïences européennes et de la porcelaine chinoise, les ateliers de potiers se développent progressivement. Ils se consacrent à la production de céramiques en pâtes claires et à glaçure stannifère où le « bleu et le blanc» répondent à un véritable modèle dans le goût de l'époque. Ces ateliers ont eu tendance à se fixer dans les quartiers de potiers de l'époque médiévale implantés à proximité des carrières d'extraction de l'argile. Il s'agit notamment de la zone orientale de Lisbonne ou encore de la partie occidentale de la ville caractérisée par une urbanisation croissante et surtout une importante activité de production en lien avec l'expansion maritime portugaise (Carita 1999 ; Mangucci 1996, 159). Au cours du XVII ${ }^{e}$ siècle, cette dualité productive s'est préservée, intensifiant le nombre d'ateliers faïenciers et d'azulejos qui ont produit une grande diversité de formes et de gammes chromatiques (Mangucci 1996; Trindade 2009a ; Sebastian 2010). Néanmoins, le corpus archéologique des centres faïenciers de Lisbonne reste réduit, se limitant au four de l'actuel Largo de Jesus (Santos 2007) et à la partie occidentale de la ville qui conserve les vestiges de dépotoirs et de carrières d'extraction d'argile dans la Rue de Buenos Aires (Batalha et al. 2012, 955-956).

L'étude du mobilier portugais découvert dans le contexte portuaire de Villefranche-sur-Mer montre une cohérence chronologique remarquable, qui se concentre sur les deux derniers tiers du XVII ${ }^{e}$ siècle. Pourtant, cette conclusion soulève de nombreuses interrogations. D'une part, il est nécessaire de distinguer les chronologies de production et de consommation. Dans le premier cas, l'absence d'études sur les ateliers de faïence empêche l'attribution de chronologies plus fines. Dans le second, on observe bien souvent des intervalles de temps très allongés et des sites très divers, que ce soit en contextes terrestres de dépotoirs, d'habitats ou en contextes maritimes avec les épaves ou encore les mouillages. D'autre part, il est nécessaire de tenir compte de l'environnement socio-économique et culturel, ainsi que de l'espace géographique. En effet, les typologies mises au jour à Villefranche-sur-Mer ont été très largement diffusées. Malgré le caractère asymétrique de la recherche archéologique portugaise pour cette époque, les exemplaires tels que ceux récupérés en France dans ce port provençal apparaissent de manière récurrente dans des contextes du centre et, en particulier, dans le sud du Portugal. Cette 
diffusion spécialisée était dès lors probablement en lien avec l'implantation géographique du centre de production. Toutefois, elle dépasse clairement l'espace européen, comme l'attestent les exemplaires découverts dans l'outre-mer portugais, en dépit du fait que la recherche archéologique dans ces territoires reste encore lacunaire. Elle s'étend en particulier au Brésil ou encore dans l'Atlantique et l'océan Indien où des faïences portugaises sont fréquemment identifiées sur les épaves. Elles sont aussi attestées dans d'autres espaces européens et américains où elles sont introduites soit en tant que produit transporté par des marchands portugais, voyageant ou établis dans ces localités, soit en tant qu'objets acquis dans les ports par les populations locales ou les équipages de passage qui les diffusent dans leur sillage.

\section{LES ÉCHANGES ENTRE LA FRANCE MÉRIDIONALE ET LE PORTUGAL AU XVII SIÈCLE}

Entre la deuxième moitié du XVII ${ }^{e}$ siècle et le milieu du XVIII ${ }^{e}$ siècle, le commerce portugais vers l'Europe s'est développé principalement avec les grandes puissances de la côte ouest de l'Atlantique, dans la péninsule Ibérique, en France, aux Pays-Bas et surtout dans les Îles Britanniques, par opposition à la perte d'importance de la Méditerranée. En effet, le poids économique de la Grande-Bretagne est devenu plus important pendant cette période grâce à ses liens historiques géo-économiques et à la diminution de la puissance maritime des Pays-Bas, qui maintenait toutefois de nombreux échanges commerciaux (Meneses 2001a, 315). L'Angleterre a par ailleurs soutenu les intérêts portugais pendant la Restauration de 1640, malgré le prix élevé demandé au Portugal pour maintenir son économie marchande. Ces liens ont établi une complémentarité évidente entre ces deux économies et leur marché, avec le ravitaillement en produits alimentaires et manufacturés anglais en échange des produits issus de l'agriculture portugaise. Ce bilan économique était toutefois clairement déséquilibré en faveur des Britanniques (Serrão 1993, 101-08).

\section{Commerce maritime et échanges franco-portugais}

Depuis le $\mathrm{XVI}^{\mathrm{e}}$ siècle, la Méditerranée occidentale a entretenu des relations commerciales avec le Portugal pour l'importation de poivre. Ce commerce prend notamment de l'essor depuis Lisbonne en direction de Gênes, Venise et la Sicile (Braudel 1990, 250). Les navires portugais transportent également des cuirs, du sel, de l'alun ou encore du sucre qu'ils acheminent dans les différents ports de commerce comme Valence, Marseille et Gênes (Braudel 1990, 312). Durant le siècle suivant, le commerce entre la France et le Portugal semble régresser. Il se résume principalement à l'échange de céréales et de produits manufacturés français et de produits coloniaux portugais, d'origine notamment brésilienne (Meneses 2001b, 334). Par ailleurs, le poids écrasant des navires étrangers dans le circuit commercial portugais limite ses échanges avec l'Europe (Rau 1954, 263). La présence de navires français dans les ports portugais représente un indicateur d'analyse important pour lequel on possède des données éparses. Ces données proviennent principalement du contrôle de l'Inquisition sur les navires étrangers ancrés au Portugal alors limités à deux ports au cours de la deuxième moitié du XVII ${ }^{\mathrm{e}}$ siècle. Ainsi, à Lisbonne, 1115 entrées de navires ont été enregistrées entre 1641-1649 et 16771685 dont $43 \%$ en provenance des Pays-Bas (nombre en diminution), 36\% d'Angleterre (nombre en croissance), $13 \%$ d'Allemagne, $2 \%$ du Danemark et seulement $2 \%$ de France (4\% d'autres nationalités). Les 20 navires français enregistrés entre 1641 et 1649 étaient liés au petit commerce, notamment du blé, mais aussi du fer, du goudron, du papier et du bois. Les principaux ports d'origine étaient La Rochelle, Nantes, Bordeaux, Saint-Malo, Bayonne, Saint-Jean-de-Luz, Busnes, Saint-Martin-deRé, Ollone, Brest et uniquement le port de Toulon pour la côte méditerranéenne. À Faro, ville du sud du Portugal très proche des échanges avec l'Andalousie espagnole, 847 entrées de navires ont été identifiées entre 1619-1657 et $1663-1683$, dont $45 \%$ battant pavillon anglais, $20 \%$ hollandais, $14 \%$ français, $9 \%$ espagnol et $10 \%$ d'autres nationalités. Parmi les 120 navires français, 87 entrées ont été enregistrées entre 1619 et 1657 . En provenance des ports atlantiques, 38 navires arrivent d'Ollone, SaintMalo, Saint-Gilles et d'autres localités non spécifiées. En provenance des ports méditerranéens, 33 arrivent de La Ciotat, Marseille, Toulon avec également plusieurs entrées dont les ports de départ ne sont pas renseignés, de même que 16 autres dont l'origine reste indéterminée. Entre 1663 et 1683, on compte seulement 33 entrées dont le port de départ est plus difficile à établir, mis à part pour La Rochelle, Nantes, Saint-Malo et Marseille, observation qui met en évidence le déclin du commerce maritime à la fin du XVII ${ }^{\mathrm{e}}$ siècle (Rau 1954, 230-32 ; 250-51). En effet, dans les années précédant la guerre de Restauration, la navigation française au Portugal était inexistante, compte tenu de l'opposition contre les Habsbourg. L'indépendance portugaise a permis une approche diplomatique, promue par le cardinal de Richelieu, qui a eu des conséquences sur l'augmentation du commerce. Néanmoins, la célébration de la paix hispano-portugaise de 1668 a marqué un éloignement diplomatique qui a abouti à la réunion des partis opposés lors de la Guerre de Succession d'Espagne, exacerbé par les conflits dans l'Amérique du sud (Meneses 2001b, 170). Dans le dernier quart du $\mathrm{XVII}^{\mathrm{e}}$ siècle, le protectionnisme de Colbert (interdiction des productions coloniales portugaises) et l'augmentation de l'emporium colonial français en Amérique (plantations de sucre et de tabac dans les Antilles) ont de plus fragilisé le traité d'alliance LusoFrançaise dans un contexte de refroidissement de l'activité commerciale européenne (Serrão 1993, 101-02; Meneses 2001b, 170 ; 334).

Ce type de céramique identifié à Villefranche-surMer est très répandu dans de nombreuses parties de l'Europe et du monde. La faible quantité de fragments ainsi que le manque d'indices matériels ne permettent ni la mise en place d'une classification, ni de pouvoir constater la présence d'une cargaison de céramique 
portugaise. Il s'agit donc vraisemblablement d'un rejet suite à un tri de la cargaison pendant la relâche du navire ou d'un rejet de mobilier de bord, pratiques fréquentes dans les mouillages. Cette céramique usuelle a donc pu être acquise dans un port portugais ou durant une escale dans un port de redistribution puis embarquée pour le service de l'équipage.

Cette première approche sur la présence de céramiques portugaises en Provence, malgré un corpus réduit, témoigne toutefois des principaux types de faïences en circulation durant les deux derniers tiers du $\mathrm{XVII}^{\mathrm{e}}$ siècle au Portugal et au sein de son réseau marchand. Cette période constitue un tournant dans l'histoire de l'Europe, marquée par une croissance importante des échanges économiques atlantiques et de profondes mutations des villes portuaires et des communautés maritimes.

\section{BIBLIOGRAPHIE}

Amouric, Richez, Vallauri 1999 : AMOURIC (H.), RICHEZ (F.), VALLAURI (L.), Vingt mille pots sous les mers, Aix-en-Provence, Edisud, 1999, 197 p.

Batalha et al. 2012 : BATALHA (L.), CAMPÔA (A.), CARDOSO (G.), NETO (N.), REBELO (P.), SANTOS (R.), Vestígios de um centro produtor de faiança dos séculos XVII e XVIII: dados de uma intervenção arqueológica na Rua de Buenos Aires, $\mathrm{n}^{\circ}$ 10, Lisboa, in : TEIXEIRA (A.), BETTENCOURT (J.), éd., Velhos e Novos Mundos. Estudos de Arqueologia Moderna, vol. 1, Lisboa, CHAM, 2012, p. 951-962.

Bluteau 1712-1728 : BLUTEAU (R.), Vocabulario portuguez \& latino: aulico, anatomico, architectonico ..., Coimbra, Collegio das Artes da Companhia de Jesu, vol. 2.

Braudel 1990 : BRAUDEL (F.), La Méditerranée et le monde méditerranéen à l'époque de Philippe II, 2 : Destins collectifs et mouvements d'ensemble, Paris, Armand Colin, 1990, 800 p.

Brito, Barbosa 2012 : BRITO (S.), BARBOSA (R.), Vestígios modernos de uma intervenção de emergência na Rua Rafael Andrade (Lisboa), in : TEIXEIRA (A.), BETTENCOURT (J.), éd., Velhos e Novos Mundos. Estudos de Arqueologia Moderna, vol. 1, Lisboa, CHAM, 2012, p. 151-156.

Bugalhão et al. 2009 : BUGALHÃO (J.), FOLGADO (D.), GOMES (S.), SOUSA (M. J.), GONZÁLEZ TINTURÉ (A.), DIAS (M.-I.), PRUDÊNCIO (M.-I.), La production céramique à Lisbonne : conclusion du projet de recherche POILX, in : Actas del VIII Congreso Internacional de Cerámica Medieval, Ciudad Real, AEAM, 2009, p. 373-398.

Calado 1992 : CALADO (R.-S.), Faiança portuguesa: sua evolução até ao início do séc. XX, Lisboa, Correios de Portugal, 1992, 120 p.
Calado 2003 : CALADO (R.-S.), Faiança portuguesa da Casa Museu Guerra Junqueiro, séculos XVIIXVIII, Porto, Câmara Municipal, 2003, 112 p.

Calado, Lima 2005 : CALADO (R.-S.), LIMA (M.-G.), coord., Portuguese faience : Guide : Museu Nacional de Arte Antiga, Lisboa, IPM, 2005, 184 p.

Cardoso, Batalha 2013 : CARDOSO (G.), BATALHA (L.), Poço seiscentista no Vale de Alcântara (Santa Isabel, Lisboa), Emerita - Estudos de Arqueologia e Património Cultural, vol. 1, 2013, p. 113-140.

Carita 1999 : CARITA, H. (1999), Lisboa manuelina e a formação de modelos urbanísticos da época moderna : 1495-1521, Lisboa, Livros Horizonte, $1999,255 \mathrm{p}$.

Carreira 2005 : CARREIRA (C.), Cerâmicas Modernas do Palácio Mogo de Melo de Torres Novas, Torres Novas, Edição Câmara Municipal de Torres Novas, 2005, $111 \mathrm{p}$.

Casimiro 2010 : CASIMIRO (T.-M.), Faiança Portuguesa nas Ilhas Britânicas (Dos Finais do século XVI aos inícios do XVIII), Lisboa, Thèse de doctorat presenté à la Faculdade de Ciências Sociais e Humanas / Universidade Nova de Lisboa, 2010.

Casimiro 2011 : CASIMIRO (T.-M.), Portuguese Faience in England and Ireland, BAR International Series 2301, Oxford, Archaeopress, 2011, 200 p.

Castro, Sebastien 2008 : CASTRO (A.-S.), SEBASTIAN (L.), A faiança portuguesa no Mosteiro de S. João de Tarouca, al-madan, $\mathrm{II}^{\mathrm{e}}$ série, $\mathrm{n}^{\mathrm{o}} 16$. Almada, Centro de Arqueologia de Almada, 2008, p. 1-32.

Dieulefet 2013 : DIEULEFET (G.), Les voies détournées du commerce en Méditerranée : constantes portuaires et commerce interlope de la mer des Baléares à la mer Tyrrhénienne ( $X V^{e}-X V I I I^{e}$ siècles). Nouveaux apports céramologiques, thèse de doctorat, Aix-Marseille Université, LA3M, 2013, 2 vol.

Dieulefet 2015 : DIEULEFET (G.), Échanges maritimes et culture matérielle : une approche par l'analyse des mouillages et des céramiques, $\mathrm{XV}^{\mathrm{e}}$-XVIII ${ }^{\mathrm{e}}$ siècles, Revue d'Histoire Maritime, numéro spécial : Les nouveaux enjeux de l'archéologie sous-marine, sous la direction de L'HOUR (M.), CÉRINO (C.), RIETH (E.), n² 21, 2015, p. 207-229.

Dieulefet, Dulière 2015 : DIEULEFET (G.), DULIÈRE (E.), Prospection archéologique sousmarine, rade de Villefranche-sur-Mer (06), Bilan scientifique $d u$ Département des recherches Archéologiques Subaquatiques et Sous-Marines, Direction Générale des Patrimoines, vol. 26, 2015, p. 84-85.

Etchevarne, Gomes 2012 : ETCHEVARNE (C.), GOMES (J.-P.), Faiança Portuguesa na Capital do Brasil Seiscentista, in : TEIXEIRA (A.), BETTENCOURT (J.), éd., Velhos e Novos Mundos. Estudos de Arqueologia Moderna, vol. 2, Lisboa, CHAM, 2012, p. 811-828. 
Fusco-Zambetogliris 2003 : FUSCO-ZAMBETOGLIRIS (N.), Un diálogo con la cerámica portuguesa de la colonia del Sacramento, Revista de Arqueologia Americana, 22, 2002, p. 43-62.

Gioffredo réed. 2008 : GIOFFREDO (P.), Histoire des Alpes maritimes : une histoire de Nice et des Alpes du sud des origines au $17^{e}$ siècle, Nice, Nice Musées édition, t. 3, réed. 2008, 721 p.

Gomes 2011 : GOMES (J.-P.), Faiança Portuguesa na Capital do Brasil Seiscentista, Coimbra, dissertation de master presenté à la Faculdade de Letras / Universidade de Coimbra, 2011.

Gomes et al. 2014 : GOMES (M.-V.), CASIMIRO (T.M.), GONÇALVES (J.), A late $17^{\text {th }}$-century Trade Cargo from Ponta do Leme Velho, Sal Island, Cape Verde", IJNA, 44.1, 2014, p. 160-172.

Gutierrez et al. 2012 : GUTIERREZ (J.-M.), BUSTAMANTE (M.), SÁNCHEZ (V.), BERNAL (D.), ARÉVALO (A.), La ocupación moderna del Teatro Romano de Cádiz (España) : nuevos datos a luz de las recientes intervenciones arqueológicas, in : TEIXEIRA (A.), BETTENCOURT (J.), éd., Velhos e Novos Mundos. Estudos de Arqueologia Moderna, vol. 1, Lisboa, CHAM, 2012, p. 261-272.

Lacroix, Bracq, Reymonenq 1999 : LACROIX (J.-B.), BRACQ (J.), REYMONENQ (J.-M.), Côtes et gens de mer des Alpes-Maritimes: XVI $-X X^{e}$ siècles. Catalogue d'exposition des Archives Départementales, Nice, Silvana Editoriale, 1999, 69 p.

Luna et al. 2006 : LUNA (I.), CARDOSO (G.), Nota preliminar sobre as cerâmicas provenientes do Poço dos Paços do Concelho de Torres Vedras», Actas do III Seminário do Património da Região Oeste, Cadava, Câmara Municipal do Cadaval, Associação Património Histórico, 2006, p. 99-112.

Mangucci 1996 : MANGUCCI (A.-C.), Olarias de Louça e Azulejo da Freguesia de Santos-o- Velho, dos meados do século XVI aos meados do século XVIII», Al-madan, II $^{\mathrm{a}}$ série, $\mathrm{n}^{\circ} 5$, Almada, Centro de Arqueologia de Almada, 1996, p. 155-168.

Mangucci 2007 : MANGUCCI (A.-C.), Da louça ordinária e não tão ordinária que se fazia em Lisboa, em 1767, Cenáculo. Boletim online do Museu de Évora, vol. 1, Évora, Museu de Évora, 2007, p. 1-8.

Meneses 2001a : MENESES (A. de F. de), A circulação, in : SERRÃO (J.), OLIVEIRA MARQUES (A. H. de), Nova História de Portugal, Portugal, da Paz da Restauração ao Ouro do Brasil, vol. II, Lisboa, Estampa, 2001, p. 302-348.

Meneses 2001b : MENESES (A. de F. de), A diplomacia $\mathrm{e}$ as relações internacionais, in : SERRÃO (J.), OLIVEIRA MARQUES (A. H. de), Nova História de Portugal, Portugal, da Paz da Restauração ao Ouro do Brasil, vol. II, Lisboa, Estampa, 2001, p. 148-191.

Moita 1987 : MOITA (I.), A casa dos Bicos - O sítio e o edifício - II, Revista Municipal de Lisboa, II : 19, Lisboa, Câmara Municipal, 1987, p. 15-32.
Monteiro, Pais 2003 : MONTEIRO (J.-P.), PAIS (A.-N.), Faiança Portuguesa da Fundação Carmona e Costa, Lisboa, Assírio \& Alvim, 2003, 118 p.

Oliveira 2010 : OLIVEIRA (C.), Um silo de Época Moderna na Rua dos Peixeiros (Lagos). Estudo do conjunto cerâmico, Xelb, vol. 10, Silves, Câmara Municipal de Silves, 2010, p. 821-840.

Pendery 1999 : PENDERY (S.-R.), Portuguese Tin-glazed Earthenware in Seventeenth-Century New England : A Preliminary Study, Historical Archaeology, vol. 33 (4), 1999, p. 58-77.

Piercy 1977 : PIERCY (R.), Mombasa wreck excavation. Preliminary report, 1977, IJNA, vol. 6.4, 1977, p. 331-347.

Piercy 1978 : PIERCY (R.), Mombasa wreck excavation. Second preliminary report, 1978, IJNA, vol. 7. 4, 1978, p. 301-319.

Pomey et al. 1987-1988 : POMEY (P.), LONG (L.), L'HOUR (M.), RICHEZ (F.), Le Brescou, épave Brescou 1, Gallia Information: recherches sousmarines, 1987-1988, 55 p.

Queirós 2002 : QUEIRÓS (J.), Cerâmica portuguesa e outros estudos, Lisboa, Editorial Presença, 2002, $528 \mathrm{p}$.

Rau 1954 : RAU (V.), Subsídios para o Estudo do Movimento dos Portos de Faro e Lisboa durante o século XVII, Anais, II : 5, Lisboa, Academia Portuguesa de História, 1954, p. 197-277.

Sá 2012 : SÁ (A.), Elementos para a caracterização da faiança portuguesa do século XVII : a tipologia de Pendery aplicada à realidade da Casa do Infante (Porto), in : TEIXEIRA (A.), BETTENCOURT (J.), éd., Velhos e Novos Mundos. Estudos de Arqueologia Moderna, vol. 2, Lisboa, CHAM, 2012, p. 963-974.

Sabrosa et al. 1992 : SABROSA (A.), ESPÍRITO SANTO (P.), Almada Medieval / Moderna: um projecto de investigação, Al-madam, vol. II: 1, Almada, Centro de Arqueologia de Almada, 1992, p. $5-12$.

Sandão 1985 : SANDÃO (A. de), Faiança portuguesa: séculos XVIII-XIX, Porto, Civilização, 1985, 404 p.

Santos 2007 : SANTOS (M.-J.), Largo de Jesus: contributo para a história incógnita de Lisboa antiga, Revista Portuguesa de Arqueologia, vol. 10, no 1, Lisboa, IPA, 2007, p. 381-399.

Sasson 1981 : SASSON (H.), Ceramics from the wreck of a Portuguese ship at Mombasa, Azania, vol. XVI, 1981, p. $97-130$

Sebastian 2010 : SEBASTIAN (L.), A Produção Oleira de Faiança em Portugal (séculos XVI-XVIII), Lisboa, thèse de doctorat présentée à la Faculdade de Ciências Sociais e Humanas / Universidade Nova de Lisboa, 2010.

Sebastian 2012 : SEBASTIAN (L.), Faiança portuguesa: centros produtores, matérias, técnicas de 
fabrico e critérios de distinção, in : TEIXEIRA (A.), BETTENCOURT (J.), éd., Velhos e Novos Mundos. Estudos de Arqueologia Moderna, vol. 2, Lisboa, CHAM, 2012, p. 937-951.

Serrão 1993 : SERRÃO (J.-V.), O quadro económico. Configurações estruturais e tendências de evolução, in : MATTOSO (J.), História de Portugal, vol. 4, O Antigo Regime (1620-1807), Lisboa, Círculo de Leitores, 1993, p. 71-117.

Silva 2013 : SILVA (N.-V.), Gold and Spices Route, On the World's Routes - Portuguese Faience $\left(16^{\text {th }}-18^{\text {th }}\right.$ centuries), Lisboa, IAP, 2013, p. 183-196.

Torres 2011 : TORRES (J.-B.), Quotidianos no Convento de São Francisco de Lisboa: uma análise da cerâmica vidrada, faiança portuguesa e porcelana chinesa, Lisboa, dissertation de master présenté à la Faculdade de Ciências Sociais e Humanas / Universidade Nova de Lisboa, 2011.
Trindade 2009a : TRINDADE (R.), A produção de louça no Reino de Portugal século XII aos meados do século XVI : leitura para uma visão de conjunto, Lisboa, thèse de doctorat présentée à la Faculdade de Ciências Sociais e Humanas / Universidade Nova de Lisboa, 2009.

Trindade 2009b : TRINDADE (R.), Imagens de Azul. Evidências do emprego do azul-cobalto na cerâmica tardo-medieval portuguesa, Revista de História de Arte, vol. 7, Lisboa, IHA, 2009, p. 236-263.

Trindade 2012 : TRINDADE (A.-R.), Convento Santana de Leiria : história, vivências e cultura material. (Cerâmicas dos Séculos XV a XVIII), Lisboa, dissertation de master présenté à la Faculdade de Ciências Sociais e Humanas / Universidade Nova de Lisboa, 2012. 
